\title{
THE ENDOMORPHISM RING OF THE ADDITIVE GROUP OF A RING
}

\author{
P. SCHULTZ*
}

(Received 1 March 1971)

Communicated by G. Szekeres

\section{Introduction}

One of the still unsolved problems posed by Fuchs in his well-known book "Abelian Groups" [2] is Problem 45: characterize the rings $R$ for which $R \cong \mathscr{E}\left(R^{+}\right)$. I present here a partial solution.

In the first part of the paper, several properties of $R$ which are simply due to the existence of an isomorphism onto $\mathscr{E}\left(R^{+}\right)$are deduced, and I am able to characterize $R$ in case it is torsion, completely decomposable, not reduced, finitely generated, or mixed with no elements of infinite $p$-height for all relevant primes $p$.

In the second part, the properties of the isomorphism of $R$ onto $\mathscr{E}\left(R^{+}\right)$are considered, and two essentially different approaches are required, depending on whether $R$ is, or is not, commutative. If it is, then $R$ is a relatively uncomplicated ring, and one can hope for a complete characterization, though this paper does not give one. If not, then $R$ must be a complicated ring indeed; for example, the group of units of $R$ contains a copy of every finite group. The best one can hope for in this case is either to exhibit such an $R$, or to prove its non-existence; once again, I am unable in this paper to do either.

I use the standard notation of abelian group theory, as found for example in Fuchs [2]. Sometimes group theoretic properties are assigned to rings; this means that the additive group of the ring has the property. For example, a ring $R$ is called torsion-free if $R^{+}$is torsion-free. Some notation which may not be familiar:

If $x \in R$, then $h(x)(p)$ means the p-height of $x$ in $R^{+}$;

$t(R)$ means the torsion subgroup of $R^{+}$;

$R_{p}$ means the p-primary component of $R^{+}$.

*Parts of this paper appear in the author's Ph.D. Thesis, University of Washington, 1968, which was written under the direction of Professor R. Beaumont. 
If $S$ is a set of primes, $S$-pure means $p$-pure for all $p \in S$, and

$S$-divisible means $p$-divisible for all $p \in S$.

$\oplus$ and $\oplus *$ mean respectively direct sum and direct product, either group or ring theoretic.

$Z, Q, Z_{p}, C(n)$ mean the group or ring (depending on context) of integers, rationals, $p$-adic integers, and integers modulo $n$.

$c$ is the cardinality of the continuum.

\section{General remarks on rings $\mathbf{R} \cong \mathscr{E}\left(\mathbf{R}^{+}\right)$}

Using some well-known invariants of abelian groups, we first characterize the divisible and torsion subgroups of such rings.

Lemma 1. Let $R \cong \mathscr{E}\left(R^{+}\right)$, and suppose $R^{+}=A \oplus D$, where $A$ is reduced and $D$ is divisible. Then either $D=0$, or $D \cong Q$ and $A$ is torsion.

Proof. The rank $m$ of the maximal torsion-free direct summand of $D$ is an invariant of $R$. Now End $\left(R^{+}\right)$, and hence $R^{+}$has a direct summand isomorphic to End $\left(\oplus_{m} Q\right)$, which is torsion free divisible, and so contained in $D$. Hence $m=0$ or 1 .

If $D$ contains a direct summand isomorphic to $C\left(p^{\infty}\right)$, then $\operatorname{End}\left(R^{+}\right)$has a direct summand isomorphic to $\operatorname{End}\left(C\left(p^{\infty}\right)\right) \cong Z_{p}$, so $R^{+}$has a direct summand isomorphic to $\operatorname{Hom}\left(Z_{p}, C\left(p^{\infty}\right)\right)$. Now $Z_{p}$ has a factor group isomorphic to $\oplus_{c} Q$, so $\operatorname{Hom}\left(Z_{p}, C\left(p^{\infty}\right)\right)$ has a subgroup isomorphic to $\operatorname{Hom}\left(\oplus_{c} Q, C\left(p^{\infty}\right)\right)$, which is torsion-free divisible of infinite rank, contradicting the first paragraph. Hence $D \cong 0$ or $Q$.

Now suppose $D \cong Q$, and let $r$ be the torsion free rank of $R^{+}$. Then End $\left(R^{+}\right)$ has a direct summand isomorphic to $\operatorname{Hom}\left(R^{+}, Q\right) \cong \oplus_{r}^{*} Q$, so $r=1$, and hence $A$ is torsion.

LEMMA 2. If $R \cong \mathscr{E}\left(R^{+}\right)$, then for each prime $p, R_{p} \cong C\left(p^{k p}\right)$ for some $0 \leqq k_{p}<\infty$.

Proof. If $R_{p} \neq 0$, it is reduced by Lemma 1 . The number $r_{n}$ of cyclic summands of $R_{p}$ of order $p^{n}$ is an invariant of $R$ for all positive integers $n$; let $k$ be minimal such that $r_{k} \neq 0$. Let $B=\oplus_{j \geqq k} B_{j}$ be a basic subgroup of $R_{p}$, where $B_{j} \cong$ $\oplus_{r_{j}} C\left(p^{j}\right)$. Now $B_{k}$ is a bounded pure subgroup, and hence a direct summand of $R^{+}$, so End $\left(R^{+}\right)$has a direct summand isomorphic to $\operatorname{Hom}\left(B_{k}, R^{+}\right)$. But this is a direct sum of cyclic groups of order $\leqq p^{k}$, so its rank $r \leqq r_{k}$. On the other hand, $\operatorname{Hom}\left(B_{k}, R^{+}\right)$has a subgroup isomorphic to $\operatorname{Hom}\left(B_{k}, B\right) \cong \oplus_{r k}^{*} \oplus_{u} C\left(p^{k}\right)$, where $u=\Sigma_{j \geqq k} r_{j}$. This subgroup has rank $2^{r_{k}} u \leqq r \leqq r_{k}$ if $r_{k}$ is infinite, or $r_{k} u \leqq r \leqq r_{k}$ if $r_{k}$ is finite. In either case, $0 \neq r_{k} \leqq u \leqq 1$, so $r_{k}=u=1$. Hence $r_{j}=0$ for all $j>k$ and $R_{p}=B=B_{k} \cong C\left(p^{k}\right)$. 
LEMMA 3. If $R \cong \mathscr{E}\left(R^{+}\right)$, and $R_{p} \neq 0$, then $R_{p}$ has a unique complement $R_{p}^{\prime}=\{x \in R \mid h(x)(p)=\infty\}$, which is an ideal in $R$; furthermore $\mathscr{E}\left(R_{p}^{\prime}\right) \cong R_{p}^{\prime}$.

Proof. Let $H$ be any group such that $R^{+}=R_{p} \oplus H$. If $p H \neq H$, then $\operatorname{End}\left(R^{+}\right)$ has a subgroup isomorphic to $\operatorname{Hom}\left(R_{p} \oplus H / p H, R_{p}\right)$. This is a $p$-group of rank $>1$, contradicting Lemma 2. Hence $p H=H$, so $H \subset R_{p}^{\prime}$. Conversely, let $x \in R_{p}^{\prime}$, and write $x=x_{1}+x_{2}$, where $x_{1} \in R_{p}, x_{2} \in H$. Since $h(x)(p)=\infty, x_{1}=0$, so $x \in H$.

$R_{p}^{\prime}$ is clearly an ideal, and $\operatorname{Hom}\left(R_{p}, R_{p}^{\prime}\right)=\operatorname{Hom}\left(R_{p}^{\prime}, R_{p}\right)=0$, so $\mathscr{E}\left(R^{+}\right)=$ $\mathscr{E}\left(R_{p}\right) \oplus \mathscr{E}\left(R_{p}^{\prime}\right) \cong R_{p} \oplus R_{p}^{\prime}$, so $R_{p}^{\prime} \cong \mathscr{E}\left(R_{p}^{\prime}\right)$.

LEMMA 4. Let $R \cong \mathscr{E}\left(R^{+}\right)$, let $S$ be the set of relevant primes for $R$, and let $U=\oplus_{p \in S}^{*} R_{p}$. Let $A=\{x \in R \mid h(x)(p)=\infty$ for all $p \in S\}$. Then $R$ is an extension of $A$ by a ring $T$ such that $t(R) \subset T \subset U$, and $T$ is an $S$-pure subring of $U$ containing the identity.

Proof. For each relevant prime $p$, we have by Lemma 3 a unique decomposition $R^{+}=R_{p} \oplus R_{p}^{\prime}$, where $R_{p}^{\prime}=\{x \in R \mid h(x)(p)=\infty\}$. Thus each $x \in R$ can be uniquely expressed as $x=x_{p}+x_{p}^{\prime}$, where $x_{p} \in R_{p}, x_{p}^{\prime} \in R_{p}^{\prime}$. Hence the mapping $e: R \rightarrow U$ given by $e(x)(p)=x_{p}$ is a well defined ring homomorphism with kernel $A$. Let $T$ be the image of $e$; clearly $e(1)$ is the identity of $U$, and $\left.e\right|_{\ell(R)}$ is the identity map, so $t(R) \subset T \subset U$.

If $T$ is not $p$-pure in $U$ for some $p \in S$, write $T=R_{p} \oplus T^{\prime}$; then $T^{\prime}$ is not $p$-pure in $\oplus_{q \neq p} R_{q}$, so $p T^{\prime} \neq T^{\prime}$. Hence End $\left(R^{+}\right)$has a subgroup isomorphic to $\operatorname{Hom}\left(R_{p} \oplus T^{\prime} / p T^{\prime}, R_{p}\right)$, which is a $p$-group of rank $>1$, a contradiction.

LemMA 5. Let $R \cong \mathscr{E}\left(R^{+}\right)$with $R^{+}$torsion-free and completely decomposable. Then $R$ is a direct sum of finitely many rank 1 rings of incomparable types.

Proof. Suppose $R^{+}=\oplus_{i \in I} A_{i}$ for some index set $I$, where each $A_{i}$ is a rank 1 torsion-free group. Then End $\left(R^{+}\right)$contains as a direct summand $\oplus_{i \in I}^{*}$ End $\left(A_{i}\right)$. Since $\operatorname{End}\left(R^{+}\right)$is completely decomposable and of rank $|I|, I$ is finite, and End $\left(R^{+}\right)=\oplus_{i \in I} \operatorname{End}\left(A_{i}\right)$. Hence for each $i \neq j$, Hom $\left(A_{i}, A_{j}\right)=0$, so the $A_{i}$ have incomparable types. Since the type of $\operatorname{End}\left(A_{i}\right)$ is less than the type of $A_{i}$, $A_{i} \cong \operatorname{End}\left(A_{i}\right)$ for all $i$, so $A_{i}$ is a rank 1 ring. Finally since $\operatorname{Hom}\left(A_{i}, A_{j}\right)=0$ if $i \neq j$, each $A_{\imath}$ is an ideal in $R$.

The characterizations promised in the Introduction follow from these lemmas:

THEOREM 1. If $R$ is torsion, then $R \cong \mathscr{E}\left(R^{+}\right)$if and only if $R$ is cyclic.

PROOF. It is well known that $C(n) \cong \mathscr{E}(C(n))$ for all positive integers $n$.

Conversely, if $R$ is torsion then by Lemma 2,

$$
\underset{p \in S}{\oplus} C\left(p^{k_{p}}\right) \cong R \cong \mathscr{E}\left(R^{+}\right) \cong \underset{p \in S}{\oplus} C\left(p^{k_{p}}\right)
$$


for some set $S$ of primes. Hence $S$ is finite, so $R$ is cyclic.

THEOREM 2. If $R^{+}$is completely decomposable, then $R \cong \mathscr{E}\left(R^{+}\right)$if and only if $R \cong C(n) \oplus A$, where $n$ is a non-negative integer and $A$ is a direct sum of rank 1 rings of incomparable type, and $A$ is divisible by each prime which divides $n$.

Proof. A modest calculation shows that $\mathscr{E}(C(n) \oplus A) \cong C(n) \oplus A$.

Conversely, let $R^{+}=t(R) \oplus A$, where $A$ is torsion-free and completely decomposable. Then $\operatorname{Hom}(t(R), A)=0$, and by $\operatorname{Lemma} 4, \operatorname{Hom}(A, t(R))=0$, so $R$ is the ring direct sum of its ideals $t(R)$ and $A$. Thus

$$
\mathscr{E}\left(R^{+}\right) \cong \mathscr{E}(t(R)) \oplus \mathscr{E}(A),
$$

so $t(R) \cong \mathscr{E}(t(R)), A \cong \mathscr{E}(A)$. The result now follows from Theorem 1, Lemma 5 , and Lemma 4.

THEOREM 3. If $R$ is not reduced, then $R \cong \mathscr{E}\left(R^{+}\right)$if and only if $R \cong Q \oplus C(n)$ for some non-negative integer $n$.

Proof. Certainly $\mathscr{E}(Q \oplus C(n)) \cong Q \oplus C(n)$.

Conversely, by Lemma $1, R \cong t(R) \oplus Q$ and by Lemma 2 ,

$$
t(R) \cong \underset{p \in S}{\oplus} C\left(p^{k_{P}}\right)
$$

for some set $S$ of primes. Then $\operatorname{End}\left(R^{+}\right)$contains a direct summand $\oplus_{p \in S}^{*} C\left(p^{k_{p}}\right) \oplus Q$; by Lemma 1 again, $\oplus_{p \in S}^{*} C\left(p^{k_{p}}\right)$ is torsion, so $S$ is finite. Hence $t(R) \cong C(n)$ for some $n$.

THEOREM 4. If $R^{+}$is finitely generated, then $R \cong \mathscr{E}\left(R^{+}\right)$if and only if $R \cong Z$, or $R \cong C(n)$ for some non-negative integer $n$.

Proof. By Theorem 2, $R$ is the direct sum of a cyclic ring and finitely many copies of the integers. But the torsion-free components have incomparable types, so there is at most one. Furthermore, $Z$ is not divisible by any prime, so if $R$ has a non-zero torsion subgroup, then $R$ is torsion.

THEOREM 5. If $R$ is a mixed ring, $S$ the set of relevant primes, and $R$ has no element of infinite p-height for all $p \in S$, then $R \cong \mathscr{E}\left(R^{+}\right)$if and only if:

(1) $R_{p} \cong C\left(p^{k p}\right), 0<k_{p}<\infty$ for all $p \in S$

(2) If $U$ is the ring $\oplus_{p \in S}^{*} R_{p}$, then $R$ is a subring with identity of $U$.

(3) $R$ is $S$-pure in $U$.

Proof. Suppose $R$ is a subring of $U$ satisfying (1), (2) and (3). The exact sequence of rings

$0 \rightarrow t(R) \rightarrow R \rightarrow R / t(R) \rightarrow 0$ induces an exact sequence of groups 


$$
0 \rightarrow \operatorname{Hom}\left(R^{+} / t(R), R^{+}\right) \rightarrow \operatorname{End}\left(R^{+}\right) \rightarrow \operatorname{Hom}\left(t(R), R^{+}\right) .
$$

Since $R$ is $S$-pure in $U, R / t(R)$ is $S$-pure in $U / t(R)$, and hence $S$-divisible. Since $R$ has no elements of infinite $p$-height for all $p \in S, \operatorname{Hom}(R / t(R), R)=0$. Hence End $\left(R^{+}\right)$is embedded in $\operatorname{Hom}(t(R), R)$ by the mapping $\left.f \leftrightarrow f\right|_{t(R)}$. But any $f \in \operatorname{Hom}(t(R), R)$ sends $t(R)$ into $t(R)$, and this mapping is a ring homomorphism. Hence $\mathscr{E}\left(R^{+}\right)$is embedded as a subring of $\mathscr{E}(t(R))$. Now it is well know that $\mathscr{E}(t(R))$ is the ring of all multiplications in $U$, so if $f \in \mathscr{E}\left(R^{+}\right)$, then $f$ is multiplication in $R$ by some $x \in U$; in particular, $x=f(1) \in R$. Thus the mapping $x \mapsto$ multiplication by $x$ is a ring homomorphism of $R$ onto $\mathscr{E}\left(R^{+}\right)$. The kernel is zero, since $1 \in R$.

Conversely, we have by Lemma 4 that $R^{+} \cong T$, an $S$-pure subring of $U=\oplus_{p \in S}^{*} R_{p}$ containing the identity, and $R_{p} \cong C\left(p^{k p}\right)$ by Lemma 2 .

Remarks. If $S$ is any infinite set of primes, and $k_{p}$ a positive integer for each $p \in S$, there are $c$ non-isomorphic subrings of

$$
\underset{p \in S}{\oplus} C\left(p^{k_{p}}\right) / \underset{p \in S}{\oplus} C\left(p^{k_{p}}\right),
$$

each of which is $S$-divisible. Hence there are $c$ non-isomorphic rings $R$ with fixed torsion subring $\oplus_{p \in S} C\left(p^{k_{p}}\right)$ which satisfy the hypotheses of Theorem 4 .

It is well known that if $R$ is a p-pure subring of $Z_{p}$, or an $S$-pure subring of $\oplus_{p \in S}^{*} Z_{p}$, where $S$ is any collection of primes, then $R \cong \mathscr{E}\left(R^{+}\right)$.

Other example of rings $R \cong \mathscr{E}\left(R^{+}\right)$can be constructed by noting that if $I$ is any index set, and $R_{i}, i \in I$, a collection of rings such that $R_{i} \cong \mathscr{E}\left(R_{i}^{+}\right)$and such that $\operatorname{Hom}\left(\oplus_{j \neq i}^{*} R_{j}, R_{i}\right)=0$, then

$$
\underset{i \in I}{\oplus *} R_{i} \cong \mathscr{E}\left(\underset{i \in I}{\oplus^{*}} R_{i}^{+}\right) .
$$

Finally, we have a slight improvement on Lemma 4.

THEOREM 6. Let $R$ be a mixed ring with relevant prime set $S$, and let

$$
A=\{x \in R \mid h(x)(p)=\infty \text { for all } p \in S\} .
$$

If $R \cong \mathscr{E}\left(R^{+}\right)$, then $R_{p} \cong C\left(p^{k_{p}}\right), 0<k_{p}<\infty$ for all $p \in S$, and $R$ is an extension of $A$ by a ring $T$ such that $T \cong \mathscr{E}\left(T^{+}\right)$and

$$
t(R) \subset T \subset \underset{p \in S}{\oplus} R_{p} .
$$

The extension splits if $A \cong \mathscr{E}\left(A^{+}\right)$.

Proof. By Lemma 4, it suffices to prove that $T \cong \mathscr{E}\left(T^{+}\right)$and the remark about splitting. The first statement follows from Theorem 5. If $A \cong \mathscr{E}\left(A^{+}\right)$, then $A$ has an identity $1_{A}$; multiplication by $1_{A}$ is a retraction of $R$ onto $A$. 


\section{The nature of the isomorphism: the commutative case}

Let $R$ be any ring with identity 1 . Then the mapping $f \mapsto f(1)_{L}$ is a retraction of $\mathscr{E}\left(R^{+}\right)$onto $R_{L}$, the ring of left multiplications in $R$. This mapping is a group homomorphism with kernel $K=\{f: f(1)=0\}$. Hence $\mathscr{E}\left(R^{+}\right)$is a group theoretic direct sum of the ring $R_{L}$ and the left ideal $K$, so we have the following:

Lemma 6. For a ring $R$ with identity 1 , the following statements are equivalent:

(1) $R$ is commutative and $R \cong \mathscr{E}\left(R^{+}\right)$.

(2) The mapping $x \rightarrow x_{L}$, left multiplication by $x$, is an isomorphism of $R$ onto $\mathscr{E}\left(R^{+}\right)$, with inverse $f \mapsto f(1)$.

(3) Every endomorphism of $R^{+}$is a left multiplication in $R$.

Proof. (1) $\rightarrow$ (2) By the preceding remarks, $R_{L}$, which is isomorphic to $R$, is a direct summand of End $\left(R^{+}\right)$. But a group with commutative endomorphism ring cannot have a proper isomorphic direct summand, so $R_{L}=\mathscr{E}\left(R^{+}\right)$, and the ring isomorphism $x \mapsto x_{L}$ maps $R$ onto $\mathscr{E}\left(R^{+}\right)$.

$(2) \rightarrow(3)$ is trivial.

(3) $\rightarrow$ (1) Clearly $\mathscr{E}\left(R^{+}\right)=R_{L} \cong R$. In particular, the right multiplications in $R$ are left multiplications; let $x_{R}$ be a right multiplication, and suppose $x_{R}=y_{L}$. Then $x=x_{R}(1)=y_{L}(1)=y$, so $x_{R}=x_{L}$; hence $R$ is commutative.

Definition.Let us call a ring $R$ which satisfies the conditions of Lemma 6 an $E$-ring, and the additive group of an $E$-ring an $E$-group. This definition of $E$-group coincides with that in [3], where an $E$-group was defined by condition (3). In Corollary 4 below, we see that every ring with identity over an $E$-group is an E-ring.

COROLlaRY 1. Every endomorphic image of an E-group $R^{+}$is the additive group of a principal ideal of $R$.

Corollary 2. A group direct summand of an E-ring $R$ is a ring direct summand, and hence $R^{+}$cannot be decomposed as an infinite direct sum.

Proof. Let $p \in \mathscr{E}\left(R^{+}\right)$be the projection of $R^{+}$onto the direct summand $e R^{+}$. Then $e=p(1)$, so

$$
e^{2}=p(1)^{2}=\mathrm{e} \cdot p(1)=p(p(1))=p(1)=e .
$$

Hence $e$ is an idempotent, so $e R$ is a ring direct summand. Since $R$ has identity 1 , $R$ cannot be an infinite direct sum of ideals.

Corollary 3. If $R$ is any ring with identity, then $\mathscr{E}\left(R^{+}\right)$is commutative if and only if $R$ is an E-ring. 
Proof. If $\mathscr{E}\left(R^{+}\right)$is commutative, let $f \in \mathscr{E}\left(R^{+}\right)$. Then for all $x \in R$,

$$
f(x)=f \cdot x_{L}(1)=x_{L} \cdot f(1)=x_{R} \cdot f(1)=f(1) x,
$$

so $f=f(1)_{L}$. Condition (3) of Lemma 6 is satisfied.

The converse follows from the definition of $E$-ring.

COROLLARY 4. If $R$ is an E-ring, and $S$ any ring with identity over $R^{+}$, then $R \cong S$.

Proof. Since $\mathscr{E}\left(R^{+}\right)=\mathscr{E}\left(S^{+}\right)$is commutative, $S$ is an $E$-ring by Corollary 3 . Hence $S \cong \mathscr{E}\left(S^{+}\right) \cong R$.

Lемма 7. Let $R$ be a ring with identity 1 , and let $F$ be an isomorphism of $R$ onto $\mathscr{E}\left(R^{+}\right)$. Then for every $* \in M u l t\left(R^{+}\right)$, there exists a unique $a \in R$ such that $x * y=F(F(a)(x))(y)$ for all $x, y \in R$.

PrOOF. There is a chain of well-known group isomorphisms

$$
R^{+} \stackrel{F}{\rightarrow} \text { End }\left(R^{+}\right) \stackrel{F^{*}}{\rightarrow} \text { Hom }\left(R^{+} \text {, End }\left(R^{+}\right)\right) \rightarrow \text { Mult }\left(R^{+}\right),
$$

where for all $a \in R^{+}, a \mapsto F(a) \mapsto f_{a} \mapsto *_{a}$, where $f_{a}(x)(y)=F(F(a)(x))(y)=x *_{a} y$.

NotE. The left multiplications in $R$ correspond to elements of $\operatorname{Mult}\left(R^{+}\right)$of the form $x * y=F(a x)(y)$ for some $a \in R$, and in particular, the identity map on $R^{+}$corresponds to the multiplication $x *_{1} y=F(x)(y)$. Clearly 1 is a left identity for $*_{1}$, and we have the following criteria for commutativity of $R$ :

LEMMA 8. Let $R$ be a ring with identity 1 , let $F$ be an isomorphism of $R$ onto $\mathscr{E}\left(R^{+}\right)$; let $a \rightarrow *_{a}$ be the mapping defined in Lemma 7. Then the following conditions are equivalent:

(1) $R$ is an E-ring

(2) Every multiplication $*_{a}$ is associative

(3) Every multiplication $*_{a}$ is commutative

(4) $*_{1}$ is associative

(5) $*_{1}$ is commutative

(6) 1 is a right identity for $*_{1}$.

PRoOF. (1) $\rightarrow(2)$ and (3).

Since $R$ is an $E$-ring, we may replace $F$, if necessary, by the isomorphism $x \rightarrow x_{L}$. Then every multiplication in $R^{+}$has the form $x *_{a} y=a x y$, so is necessarily associative and commutative.

$(2) \rightarrow(4)$ and $(3) \rightarrow(5)$ are trivial

(4) $\rightarrow$ (6) For all $x, y, z \in R$,

$$
F(F(x)(y))(z)=\left(x_{1} * y\right) *_{1} z=x *_{1}\left(y *_{1} z\right)=F(x)(F(y)(z))=F(x y)(z),
$$


so $F\left(F(x)(y)=F(x y)\right.$. Hence $x *_{1} y=F(x)(y)=x y$ for all $x, y \in R$ and 1 is a right identity.

$(5) \rightarrow(6)$ For all $x \in R, x *_{1} 1=1 *_{1} x=F(1)(x)=x$.

(6) $\rightarrow$ (1) If $x=x *_{1} 1=F(x)$ (1) for all $x$, then

$$
K=\{f \in \mathscr{E}(R) \mid f(1)=0\}=0,
$$

so by the Remarks preceding Lemma $6, \mathscr{E}\left(R^{+}\right)=R_{L}$ and $R$ is an $E$-ring.

COROLlaRY 5. If $R$ is an E-ring, there is a 1-1 correspondence between elements a of $R$, and rings $R_{a}$ over $R^{+}$. This correspondence maps 1 into $R$, and units $u$ of $R$ into rings $R_{u}$ with identity $u^{-1}$ such that $R_{u} \cong R$.

Proof. By Lemma 8 , every element $*_{a}$ of $\operatorname{Mult}\left(R^{+}\right)$is associative, and so defines a ring $R_{a}$ over $R^{+}$, whose multiplication is given by $x * y=a x y$; since every ring $R^{\prime}$ over $R^{+}$gives rise to some multiplication in $R^{+}$, this correspondence is 1-1. The original ring $R=R_{1}$, and clearly $u^{-1}$ is an identity for $R_{u}$. Of course all rings with identity over $R^{+}$are isomorphic by Corollary 4 .

LemMa 9. Any endomorphic image of an E-group is an E-group; every endomorphism of an endomorphic image of an E-group $R^{+}$can be extended to an endomorphism of $R^{+}$.

Proof. If $R$ is an $E$-ring, any endomorphic image of $R^{+}$has the form $a R^{+}$ f $s$ r some $a \in R$ by Corollary 1 . Define a multiplication $*$ on $a R^{+}$by $a x * a y=a x y$. Then $*$ induces a commutative ring $S$ over $a R^{+}$with identity $a$. Let $f \in \mathscr{E}\left(a R^{+}\right)$; then $f \cdot a_{L} \in \mathscr{E}\left(R^{+}\right)$, so for all $a x \in a R$,

$$
f(a x)=\left(f \cdot a_{L}\right)(1) \cdot x=f(a) \cdot x=a y x,
$$

where $a y=f(a) \in a R^{+}$. Hence $f(a x)=a y * a x$, so $f$ is multiplication by $a y$ in $S$. By Lemma 6, $S$ is an $E$-ring, and $a R^{+}$an $E$-group.

Let $f \in \mathscr{E}\left(a R^{+}\right)$, say $f(a)=a y$. Then $y_{L} \in \mathscr{E}\left(R^{+}\right)$is an extension of $f$, for if $a x \in a R$,

$$
f(a x)=a y * a x=a x y .
$$

Unfortunately, no such nice property seems to be true in general for rings $R \cong \mathscr{E}\left(R^{+}\right)$. We do know that $\mathscr{E}\left(R^{+}\right)=R_{L} \oplus K$, where $K=\{f \mid f(1)=0\}$. If $R$ is not commutative, then by Lemma $5, K \neq 0$, so $R^{+}$is isomorphic to a proper direct summand, the inverse image of $R_{L}$. Clearly, none of the rings described in Theorem 1-5 have this property, so they are all $E$-rings. In addition, we have the following partial result, corresponding to Theorem 6 .

THEOREM 7. Let $R$ be a mixed ring with identity with a set $S$ of relevant primes. Let 


$$
A=\{x \in R \mid h(x)(p)=\infty \text { for all } p \in S\},
$$

and let $U=\oplus_{p \in S}^{*} R_{p}$. Then $R$ is an E-ring if and only if:

(1) $R_{p} \cong\left(C p^{k p}\right), 0<k_{p}<\infty$ for all $p \in S$

(2) $R$ is an extension of $A$ by an E-ring $T$ such that $t(R) \subset T \subset U$

(3) If $f \in \mathscr{E}\left(R^{+}\right)$, then the restriction of $f-f(1)_{L}$ to $A$ is the zero map.

(4) If $f \in \mathscr{E}\left(R^{+}\right)$, then the unique homomorphism $\bar{f}: T / t(R) \rightarrow A$ induced by $f-f(1)_{L}$ is the zero map.

Proof. Suppose $R$ is an $E$-ring. Condition (1) follows from Lemma 2. By Theorem $6, R$ is an extension of $A$ by a ring $T \cong \mathscr{E}\left(T^{+}\right)$such that $t(R) \subset T \subset U$. Since $T$ is commutative, it is an $E$-ring. Conditions (3) and (4) are trivial, since $=f(1)_{L}$.

Now assume that $R$ satisfies conditions (1)-(4), let $f \in \mathscr{E}\left(R^{+}\right)$, and consider the endomorphism $f^{\prime}=f-f(1)_{L}$. Since $\left.f^{\prime}\right|_{R_{p}} \in \mathscr{E}\left(R_{p}\right)$, it is multiplication in $R_{p}$ by $f^{\prime}\left(1_{p}\right)$, where $1_{p}$, the $p$-component of 1 , is the identity of $R_{p}$. Hence $f^{\prime}\left(1_{p}\right)=0$ for all $p \in S$, so $\left.f^{\prime}\right|_{t(R)}=0$. By condition (3), $\left.f^{\prime}\right|_{A}=0$, so $A \oplus t(R)$ is contained in the kernel of $f^{\prime}$; thus $f^{\prime}$ induces a unique homorphism $\bar{f}$ on

$$
R^{+} / A \oplus t(R) \cong T / t(R)
$$

whose image must be a subgroup of $A$, since $T / t(R)$ is $S$-divisible. Hence by condition (4) $\bar{f}=0$ so $f=f(1)_{L}$. Thus $R$ is an $E$-ring.

Remark. Conditions (3) and (4) are of little use in either constructing $E$ rings, or deciding whether a ring is an $E$-ring. It might be conjectured that they could be replaced by stronger conditions, for example:

(3') $A$ is an E-ring

(4') $\operatorname{Hom}(T, A)=0$.

However, ( $\left.3^{\prime}\right)$ is true if (by Theorem 6) and only if (since $\mathscr{E}\left(R^{+}\right)$is commutative) the extension of Theorem 7 splits, so $\left(3^{\prime}\right)$ implies $\left(4^{\prime}\right)$. While no non-splitting extension has been constructed, there seems no reason to believe they do not exist. Condition ( $\left.4^{\prime}\right)$ seems more reasonable, and I conjecture that it is a necessary condition for $R$ to be an $E$-ring.

\section{The nature of the isomorphism: the non-commutative case}

Let $R \cong \mathscr{E}\left(R^{+}\right)$, with $R$ not commutative. Then we know from the remarks preceding Lemma 6 that:

$$
\mathscr{E}\left(R^{+}\right)=R_{L} \oplus K, \text { where } K=\{f \in \mathscr{E}(R) \mid f(1)=0\} \neq 0 .
$$

Hence End $\left(R^{+}\right)$, and consequently $R^{+}$, is an $I D$-group, that is, a group which is isomorphic to a proper direct summand. (It is not difficult to find elements of $K$ : for example, if $x$ is not in the centre of $R$, then $\left.x_{L}-x_{R} \in K\right)$. Now if $R^{+}$is an 
$I D$-group, there exist monomorphisms $\phi \in \mathscr{E}\left(R^{+}\right)$such that $R^{+}=\phi\left(R^{+}\right) \oplus H$, where $H \neq 0$. Beaumont and Pierce [1] have proved the following structure theorem for $I D$-groups $R^{+}$:

Let $M=\bigcap_{n<\omega} \phi^{n}\left(R^{+}\right)$, let $P=\oplus_{n<\omega}^{*} H_{n}$, where $H_{n}=\phi^{n}(H) \cong H$, and let $S=\Theta_{n<\omega} H_{n}$. Then $R^{+}$is an extension of $M$ by a group $T$ such that $S \subset T \subset P$, and $\phi \mid M$ is an automorphism of $M$.

In our case, let $F: R \rightarrow \mathscr{E}\left(R^{+}\right)$be the isomorphism, and define $\phi$ by $\phi(x)$ $=F^{-1}\left(x_{L}\right)$ for $x \in R^{+}$. Then $F\left(\phi\left(R^{+}\right)\right)=R_{L}$ so $F(H)=K$ and by Beaumonts and Pierce's result, $R^{+}$is an extension of $\bigcap_{n<\omega} \varphi^{n}\left(R^{+}\right)$by a subgroup of $\oplus_{x_{0}}^{*} K$. If $R$ contains a fully invariant $E$-ring $A$, then clearly $F$ can be modified so that $F(x)=x_{L}$ for $x \in A$, and in this case, $\left.\phi\right|_{A}$ is the identity, so $A^{+} \subset \bigcap_{n<\omega} \phi^{n}\left(R^{+}\right)$. In general however, little else is known.

LEMMA 10. Let $R \cong \mathscr{E}\left(R^{+}\right), R$ not commutative. Then the group $U$ of units of $R$ contains a copy of every finite group.

Proof. It will suffice to show that $U$ contains a copy of $S(n)$, the symmetric group on $n$ symbols for every positive integer $n$. Now

$$
R^{+}=H \oplus \phi(H) \oplus \cdots_{\oplus} \phi^{n-1}(H) \oplus \phi^{n}\left(R^{+}\right),
$$

where $\phi^{i}(H) \cong H$, so each $x \in R^{+}$can be expressed as

$$
x=\left(x_{1}, \phi\left(x_{2}\right), \cdots, \phi^{n-1}\left(x_{n}\right), y\right),
$$

with $x_{i} \in H$ for $i=1,2, \cdots n$. For $\sigma \in S(n)$, define $f_{\sigma} \in \mathscr{E}\left(R^{+}\right)$by

$$
f_{\sigma}\left(x_{1}, \phi\left(x_{2}\right), \cdots, \phi^{n-1}\left(x_{n}\right), y\right)=\left(x_{\sigma(1)}, \phi\left(x_{\sigma(2)}\right), \cdots, \phi^{n-1}\left(x_{\sigma(n)}\right), y\right) .
$$

$f_{\sigma}$ is clearly an automorphism of $R^{+}$, so the set $\left\{f_{\sigma}: \sigma \in S(n)\right\}$ is a group of units of $\mathscr{E}\left(R^{+}\right)$isomorphic to $S(n)$.

\section{References}

[1] R. A. Beaumont and R. S. Pierce, 'Isomorphic Direct Summands of Abelian Groups', Math. Annalen 153 (1964), 21-37.

[2] L. Fuchs, Abelian Groups (Hungarian Academy of Science, Budapest, 1958).

[3] P. Schultz, 'Periodic Homomorphism Sequences of Abelian Groups', Arch. Math. 21 (1970), 132-135.

Department of Mathematics

University of Western Australia 